\title{
Application of Local Fractional Variational Iteration Method for Solving Fredholm integral equations Involving Local Fractional Operators \\ https://doi.org/10.32792/utq/utj/vol11/1/2
}

\author{
Hassan Kamil Jassim $^{1}$ and Hussein Khashan Kadhim ${ }^{2}$ \\ Department of Mathematics, Faculty of Education for Pure Sciences, University \\ of Thi-Qar, Nasiriyah, Iraq \\ hassan.kamil@yahoo.com and ${ }^{2}$ kadhimhussein82@yahoo.com
}

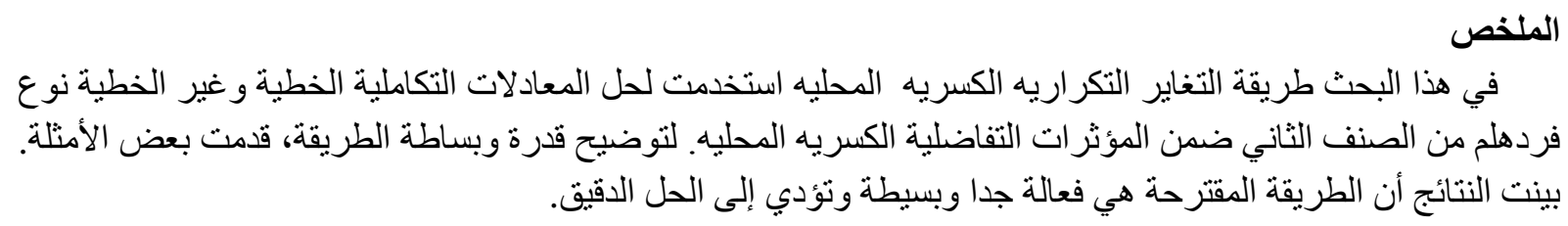

\begin{abstract}
In this paper, the local fractional variational iteration method (LFVIM) is used for solving linear and nonlinear Fredholm integral equations of the second kind within local fractional derivative operators. To illustrate the ability and simplicity of the method, some examples are provided. The results reveal that the proposed method is very effective and simple and it leads to the exact solution.
\end{abstract}

Keywords: Linear and nonlinear Fredholm integral equation, Local fractional variational Iteration Method, Local fractional operator.

\section{Introduction}

Integral equation is encountered in a variety of applications in many fields including continuum mechanics, potential theory, geophysics, electricity and magnetism, kinetic theory of gases, hereditary phenomena in physics and biology, renewal theory, quantum mechanics, radiation, optimization, optimal control systems, communication theory, mathematical economics, population genetics, queuing theory, medicine, mathematical problems of radioactive equilibrium, the particle transport problems of astrophysics and reactor theory, acoustics, fluid mechanics, steady state heat conduction, and radioactive heat transfer problems. Fredholm integral equation is one of the most important integral equations (Ray and Sahu, 2013 and Wazwaz, 2011).

The theory of local fractional derivatives and integrals was dealing with fractal functions, and was successfully used in local fractional Fokker Planck equation (Kolwankar and Gangal,1997 and Kolwankar and Gangal,1998), local fractional diffusion equation (Fan et al, 2015, Xu et al, 2015), local fractional integral equations (Yang (11th reference), 2011), local fractional ordinary differential equations (Yang (10th reference), 2011 and Yang (11th 


\section{University of Thi-Qar Journal Vol.11 No.1 Mar 2016}

Web Site: https://jutq.utq.edu.iq/index.php/main Email: journal@jutq.utq.edu.iq

reference), 2011), local fractional partial differential equation (Jassim , 2015, Wang et al, 2014, Yan et al, 2014 and Yang, 2012). In this paper, we investigate the application of local fractional variational iteration methods for solving the local fractional Fredholm integral equations of the second kind. The general form of this integral equation is given by

$$
u(x)=f(x)+\frac{1}{\Gamma(1+\alpha)} \int_{a}^{b} K(x, t) F(u(t))(d t)^{\alpha}, 0<\alpha \leq 1, a \leq x \leq b
$$

where $k(x, t)$ is the kernel of the local fractional integral equation, $f(x)$ and $F(u)$ are known functions. The limits of integration $a$ and $b$ are constants and $u(x)$ is the unknown solution of integral equation, which we are going to find, via local fractional variational iteration method. The paper has been organized as follows. In Section 2, we give the concept of local fractional calculus. In Section 3, we give analysis of the methods used. In Section 4, we consider several illustrative examples. Finally, in Section 5, we present our conclusions.

\section{Preliminaries}

In this section we present some basic definitions and notations of the local fractional operators (see(Wang et al, 2014,Yan et al, 2014, Yang (10th reference), 2011 and Yang, 2012)).

Definition 1. The local fractional derivative of $\psi(x)$ of order $\alpha$ at $x=x_{0}$ is given by

$$
\left.\frac{d^{\alpha}}{d x^{\alpha}} \psi(x)\right|_{x=x_{0}}=\psi^{(\alpha)}\left(x_{0}\right)=\lim _{x \rightarrow x_{0}} \frac{\Delta^{\alpha}\left(\psi(x)-\psi\left(x_{0}\right)\right)}{\left(x-x_{0}\right)^{\alpha}},
$$

where $\Delta^{\alpha}\left(\psi(x)-\psi\left(x_{0}\right)\right) \cong \Gamma(\alpha+1)\left(\psi(x)-\psi\left(x_{0}\right)\right)$.

The formulas of local fractional derivatives of special functions used in the paper are as follows:

$$
\begin{aligned}
& D_{x}^{(\alpha)} a \psi(x)=a D_{x}^{(\alpha)} \psi(x), \\
& \frac{d^{\alpha}}{d x^{\alpha}}\left(\frac{x^{n \alpha}}{\Gamma(1+n \alpha)}\right)=\frac{x^{(n-1)^{\alpha}}}{\Gamma(1+(n-1) \alpha)}, n \in N
\end{aligned}
$$

Definition 2. The local fractional integral of $\psi(x)$ of order $\alpha$ in the interval $[a, b]$ is given by

$$
{ }_{a} I_{b}^{(\alpha)} \psi(x)=\frac{1}{\Gamma(1+\alpha)} \int_{a}^{b} \psi(t)(d t)^{\alpha}=\frac{1}{\Gamma(1+\alpha)} \lim _{\Delta t \rightarrow 0} \sum_{j=0}^{N-1} \psi\left(t_{j}\right)\left(\Delta t_{j}\right)^{\alpha},
$$

where the partitions of the interval $[a, b]$ are denoted as $\left(t_{j}, t_{j+1}\right)$, with $\Delta t_{j}=t_{j+1}-t_{j}, t_{0}=a$, $t_{N}=b$ and $\Delta t=\max \left\{\Delta t_{0}, \Delta t_{1}, \ldots ..\right\}, j=0, \ldots, N-1$.

The formulas of local fractional integrals of special functions used in the paper are as follows:

$$
\begin{aligned}
& { }_{0} I_{x}^{(\alpha)} a \psi(x)=a_{0} I_{x}^{(\alpha)} \psi(x), \\
& { }_{0} I_{x}^{(\alpha)}\left(\frac{x^{n \alpha}}{\Gamma(1+n \alpha)}\right)=\frac{x^{(n+1)^{\alpha}}}{\Gamma(1+(n+1) \alpha)}, n \in N
\end{aligned}
$$




\section{University of Thi-Qar Journal Vol.11 No.1 Mar 2016}

Web Site: https://jutq.utq.edu.iq/index.php/main Email: journal@jutq.utq.edu.iq

Definition 3. The Mittage Leffler function is defined as

$$
E_{\alpha}\left(x^{\alpha}\right)=\sum_{k=0}^{\infty} \frac{x^{k \alpha}}{\Gamma(1+k \alpha)}, 0<\alpha \leq 1
$$

\section{Analysis of the Method}

For solving equation (1.1) by local fractional variational iteration method, first we differentiate once from both sides of equation (1.1) with respect to $x$ :

$$
u^{(\alpha)}(x)=f^{(\alpha)}(x)+\frac{1}{\Gamma(1+\alpha)} \int_{a}^{b} \frac{\partial^{\alpha} K(x, t)}{\partial x^{\alpha}} F(u(t))(d t)^{\alpha}
$$

Now, we apply local fractional variational iteration method for equation (3.1). According to this method correction functional can be written in the following form (Xu et al, 2015) :

$$
u_{n+1}(x)=u_{n}(x)+{ }_{0} I_{x}^{(\alpha)}\left(\frac{\lambda(\zeta)^{\alpha}}{\Gamma(1+\alpha)}\left[u_{n}^{(\alpha)}(\zeta)-f^{(\alpha)}(\zeta)-\frac{1}{\Gamma(1+\alpha)} \int_{a}^{b} \frac{\partial^{\alpha} K(\xi, t)}{\partial \xi^{\alpha}} F\left(\tilde{u}_{n}(t)\right)(d t)^{\alpha}\right]\right) \text {, }
$$

where $\frac{\lambda(\zeta)^{\alpha}}{\Gamma(1+\alpha)}$ is a general fractal Lagrange's multiplier. To make the above correction functional stationary with respect to $u_{n}$, we have :

$$
\begin{aligned}
\delta^{\alpha} u_{n+1}(x) & =\delta^{\alpha} u_{n}(x)+\delta^{\alpha}{ }_{0} I_{x}^{(\alpha)}\left(\frac{\lambda(\zeta)^{\alpha}}{\Gamma(1+\alpha)}\left[u_{n}{ }^{(\alpha)}(\zeta)-f^{(\alpha)}(\zeta)-\frac{1}{\Gamma(1+\alpha)} \int_{a}^{b} \frac{\partial^{\alpha} K(\xi, t)}{\partial \xi^{\alpha}} F\left(\tilde{u}_{n}(t)\right)(d t)^{\alpha}\right]\right) \\
& =\delta^{\alpha} u_{n}(x)+{ }_{0} I_{x}^{(\alpha)}\left(\frac{\lambda(\zeta)^{\alpha}}{\Gamma(1+\alpha)} \delta^{\alpha}\left(u_{n}^{(\alpha)}(\xi)\right)\right) \\
& =\delta^{\alpha} u_{n}(x)+\frac{\lambda(x)^{\alpha}}{\Gamma(1+\alpha)} \delta^{\alpha} u_{n}(x)+{ }_{0} I_{x}^{(\alpha)}\left(\frac{\lambda^{(\alpha)}(\zeta)^{\alpha}}{\Gamma(1+\alpha)} \delta^{\alpha} u_{n}(\xi)\right)=0 .
\end{aligned}
$$

From the above relation for any $\delta^{\alpha} u_{n}$ (variation of $u_{n}$ ), we obtain

$$
1+\left.\frac{\lambda_{i}(\xi)^{\alpha}}{\Gamma(1+\alpha)}\right|_{\xi=t}=0,\left.\left(\frac{\lambda_{i}(\xi)^{\alpha}}{\Gamma(1+\alpha)}\right)^{(\alpha)}\right|_{\xi=t}=0
$$

This in turn gives

$$
\frac{\lambda(\zeta)^{\alpha}}{\Gamma(1+\alpha)}=-1
$$

Substituting the identified Lagrange multiplier (3.3) into equation (3.2), result in the following iterative formula:

$$
u_{n+1}(x)=u_{n}(x)-{ }_{0} I_{x}^{(\alpha)}\left(u_{n}^{(\alpha)}(\xi)-f^{(\alpha)}(\xi)-\frac{1}{\Gamma(1+\alpha)} \int_{a}^{b} \frac{\partial^{\alpha} K(\xi, t)}{\partial \xi^{\alpha}} F\left(u_{n}(t)\right)(d t)^{\alpha}\right) .
$$

Finally, we obtain the exact solution or an approximate solution of the equation (1.1) as follows: 


\section{University of Thi-Qar Journal Vol.11 No.1 Mar 2016}

Web Site: https://jutq.utq.edu.iq/index.php/main Email: journal@jutq.utq.edu.iq

$$
u(x, t)=\lim _{n \rightarrow \infty} u_{n}(x, t)
$$

\section{Illustrative examples}

To illustrate the ability and simplicity of the proposed method, some examples are provided here.

Example 1. Consider the following linear local fractional Fredholm integral equation

$$
u(x)=E_{\alpha}\left(x^{\alpha}\right)-\frac{x^{\alpha}}{\Gamma(1+\alpha)}+\frac{1}{\Gamma(1+\alpha)} \int_{0}^{1} \frac{x^{\alpha}}{\Gamma(1+\alpha)} \frac{t^{\alpha}}{\Gamma(1+\alpha)} u(t)(d t)^{\alpha},
$$

with the exact solution, $u(x)=E_{\alpha}\left(x^{\alpha}\right)$.

Differentiating both sides Eq. (4.1) with respect to $x$ yields

$$
u^{(\alpha)}(x)=E_{\alpha}\left(x^{\alpha}\right)-1+\frac{1}{\Gamma(1+\alpha)} \int_{0}^{1} \frac{t^{\alpha}}{\Gamma(1+\alpha)} u(t)(d t)^{\alpha}
$$

The correction functional for (4.2) is given by

$$
u_{n+1}(x)=u_{n}(x)-{ }_{0} I_{x}^{(\alpha)}\left(u_{n}^{(\alpha)}(\zeta)-E_{\alpha}\left(\zeta^{\alpha}\right)+1-\frac{1}{\Gamma(1+\alpha)} \int_{0}^{1} \frac{r^{\alpha}}{\Gamma(1+\alpha)} u_{n}(r)(d r)^{\alpha}\right)
$$

when we put $\frac{\lambda(\zeta)^{\alpha}}{\Gamma(1+\alpha)}=-1$.

Notice that the initial condition $u(0)=1$ is obtained by substituting $x=0$ into (4.1).

Therefore, we have

$$
\begin{aligned}
u_{0}(x) & =1 \\
u_{1}(x) & =u_{0}(x)-{ }_{0} I_{x}^{(\alpha)}\left(u_{0}^{(\alpha)}(\zeta)-E_{\alpha}\left(\zeta^{\alpha}\right)+1-\frac{1}{\Gamma(1+\alpha)} \int_{0}^{1} \frac{r^{\alpha}}{\Gamma(1+\alpha)} u_{0}(r)(d r)^{\alpha}\right) \\
& =E_{\alpha}\left(x^{\alpha}\right)-\frac{1}{2} \frac{x^{\alpha}}{\Gamma(1+\alpha)} \\
u_{2}(x) & =u_{1}(x)-{ }_{0} I_{x}^{(\alpha)}\left(u_{1}^{(\alpha)}(\zeta)-E_{\alpha}\left(\zeta^{\alpha}\right)+1-\frac{1}{\Gamma(1+\alpha)} \int_{0}^{1} \frac{r^{\alpha}}{\Gamma(1+\alpha)} u_{1}(r)(d r)^{\alpha}\right) \\
& =E_{\alpha}\left(x^{\alpha}\right)-\frac{1}{6} \frac{x^{\alpha}}{\Gamma(1+\alpha)} \\
u_{3}(x) & =u_{2}(x)-{ }_{0} I_{x}^{(\alpha)}\left(u_{2}^{(\alpha)}(\zeta)-E_{\alpha}\left(\zeta^{\alpha}\right)+1-\frac{1}{\Gamma(1+\alpha)} \int_{0}^{1} \frac{r^{\alpha}}{\Gamma(1+\alpha)} u_{2}(r)(d r)^{\alpha}\right) \\
& =E_{\alpha}\left(x^{\alpha}\right)-\frac{1}{18} \frac{x^{\alpha}}{\Gamma(1+\alpha)}
\end{aligned}
$$


Web Site: https://jutq.utq.edu.iq/index.php/main Email: journal@jutq.utq.edu.iq

$$
u_{n}(x)=E_{\alpha}\left(x^{\alpha}\right)-\frac{1}{2 \times 3^{n-1}} \frac{x^{\alpha}}{\Gamma(1+\alpha)}, n \geq 1 .
$$

Thus, we have

$$
\begin{aligned}
u(x) & =\lim _{n \rightarrow \infty} u_{n}(x) \\
& =\lim _{n \rightarrow \infty}\left[E_{\alpha}\left(x^{\alpha}\right)-\frac{1}{2 \times 3^{n-1}} \frac{x^{\alpha}}{\Gamma(1+\alpha)}\right]=E_{\alpha}\left(x^{\alpha}\right),
\end{aligned}
$$

which is the exact solution.

Example 2. Consider the following nonlinear local fractional Fredholm integral equation

$$
u(x)=\frac{7}{8} \frac{x^{\alpha}}{\Gamma(1+\alpha)}+\frac{1}{2} \frac{1}{\Gamma(1+\alpha)} \int_{0}^{1} \frac{x^{\alpha}}{\Gamma(1+\alpha)} \frac{t^{\alpha}}{\Gamma(1+\alpha)} u^{2}(t)(d t)^{\alpha},
$$

with the exact solution, $u(x)=\frac{x^{\alpha}}{\Gamma(1+\alpha)}$.

In view (3.4) and (4.9), the local fractional iteration algorithm can be written as follows:

$$
u_{n+1}(x)=u_{n}(x)-{ }_{0} I_{x}^{(\alpha)}\left(u_{n}^{(\alpha)}(\zeta)-\frac{7}{8}+\frac{1}{2} \frac{1}{\Gamma(1+\alpha)} \int_{0}^{1} \frac{t^{\alpha}}{\Gamma(1+\alpha)} u_{n}^{2}(t)(d t)^{\alpha}\right) \text {. }
$$

Consider the initial approximation $u_{0}(x)=\frac{7}{8} \frac{x^{\alpha}}{\Gamma(1+\alpha)}$.

Therefore other terms of the sequence are computed as follows:

$$
\begin{aligned}
u_{0}(x) & =\frac{7}{8} \frac{x^{\alpha}}{\Gamma(1+\alpha)}, \\
u_{1}(x) & =u_{0}(x)-{ }_{0} I_{x}^{(\alpha)}\left(u_{0}^{(\alpha)}(\zeta)-\frac{7}{8}+\frac{1}{2} \frac{1}{\Gamma(1+\alpha)} \int_{0}^{1} \frac{t^{\alpha}}{\Gamma(1+\alpha)} u_{0}^{2}(t)(d t)^{\alpha}\right) \\
& =\frac{497}{512} \frac{x^{\alpha}}{\Gamma(1+\alpha)}, \\
u_{2}(x) & =u_{1}(x)-{ }_{0} I_{x}^{(\alpha)}\left(u_{1}^{(\alpha)}(\zeta)-\frac{7}{8}+\frac{1}{2} \frac{1}{\Gamma(1+\alpha)} \int_{0}^{1} \frac{t^{\alpha}}{\Gamma(1+\alpha)} u_{1}^{2}(t)(d t)^{\alpha}\right) \\
& =\frac{2082017}{2097152} \frac{x^{\alpha}}{\Gamma(1+\alpha)},
\end{aligned}
$$

and so on. The sequence tends to $\frac{x^{\alpha}}{\Gamma(1+\alpha)}$ as $n \rightarrow \infty$, which is the exact solution.

Example 3. Consider the following nonlinear local fractional Fredholm integral equation

$$
u(x)=\cos _{\alpha}\left(x^{\alpha}\right)-\frac{x^{\alpha}}{\Gamma(1+\alpha)}+\frac{1}{\Gamma(1+\alpha)} \int_{0}^{1} \frac{x^{\alpha}}{\Gamma(1+\alpha)}\left(u^{2}(t)+\sin _{\alpha}^{2}\left(t^{\alpha}\right)\right)(d t)^{\alpha},
$$

with the exact solution, $u(x)=\cos _{\alpha}\left(x^{\alpha}\right)$.

In the same procedure, the iterative formula can be expressed as the following: 


$$
u_{n+1}(x)=u_{n}(x)-{ }_{0} I_{x}^{(\alpha)}\left(u_{n}^{(\alpha)}(\zeta)+\sin _{\alpha}\left(\xi^{\alpha}\right)+1-\frac{1}{\Gamma(1+\alpha)} \int_{0}^{1}\left(u_{n}^{2}(t)+\sin _{\alpha}^{2}(t)\right)(d t)^{\alpha}\right) .
$$

By using this iterative formula and taking $u_{0}(x)=\cos _{\alpha}\left(x^{\alpha}\right)$, we have:

$$
\begin{aligned}
& u_{0}(x)=\cos _{\alpha}\left(x^{\alpha}\right) \text {, } \\
& u_{1}(x)=u_{0}(x)-{ }_{0} I_{x}^{(\alpha)}\left(u_{0}^{(\alpha)}(\zeta)+\sin _{\alpha}\left(\xi^{\alpha}\right)+1-\frac{1}{\Gamma(1+\alpha)} \int_{0}^{1}\left(u_{0}^{2}(t)+\sin _{\alpha}^{2}(t)\right)(d t)^{\alpha}\right)=\cos _{\alpha}\left(x^{\alpha}\right),(4 \\
& u_{2}(x)=u_{1}(x)-{ }_{0} I_{x}^{(\alpha)}\left(u_{1}^{(\alpha)}(\zeta)+\sin _{\alpha}\left(\xi^{\alpha}\right)+1-\frac{1}{\Gamma(1+\alpha)} \int_{0}^{1}\left(u_{1}^{2}(t)+\sin _{\alpha}^{2}(t)\right)(d t)^{\alpha}\right)=\cos _{\alpha}\left(x^{\alpha}\right),(4 \\
& u_{n}(x)=\cos _{\alpha}\left(x^{\alpha}\right) \text {. }
\end{aligned}
$$

Thus, we have

$$
\begin{aligned}
u(x) & =\lim _{n \rightarrow \infty} u_{n}(x) \\
& =\lim _{n \rightarrow \infty} \cos _{\alpha}\left(x^{\alpha}\right) \\
& =\cos _{\alpha}\left(x^{\alpha}\right),
\end{aligned}
$$

which is the exact solution.

\section{Conclusion}

In this work local fractional variational iteration method has been used successfully for solving the linear and nonlinear Fredholm integral equations on local fractional derivative operators. The present method reduces the computational difficulties of the other methods and all the calculations can be made simply. On the other hand the results are quite reliable. The present study has confirmed that the local fractional variational iteration method offers great advantages of straightforward applicability, computational efficiency and high accuracy.

\section{References}

Fan ,Z. P., Jassim, H. K., Rainna, R. K., and Yang, X. J.,(2015), Adomian Decomposition Method for Three-Dimensional Diffusion Model in Fractal Heat Transfer Involving Local Fractional Derivatives, Thermal Science, Vol. 19, Suppl. 1, 137-141.

Jassim ,H. K., (2015) ,Local Fractional Laplace Decomposition Method for Nonhomogeneous Heat Equations Arising in Fractal Heat Flow with Local Fractional Derivative, International Journal of Advances in Applied Mathematics and Mechanics, vol.2, no. 4 ,1-7.

Kolwankar ,K. M. and Gangal, A. D., (1997), Hölder exponents of irregular signals and local fractional derivatives, Pramana J. Phys., 48, 49-68.

Kolwankar ,K. M. and Gangal, A. D.,( 1998), Local fractional Fokker Planck equation, Phys. Rev. Lett., 80 ,( 214-217). 


\section{University of Thi-Qar Journal Vol.11 No.1 Mar 2016}

Web Site: https://jutq.utq.edu.iq/index.php/main Email: journal@jutq.utq.edu.iq

Ray ,S. S. and Sahu, P. K., (2013), Numerical Methods for Solving Fredholm Integral Equations of Second Kind, Abstract and Applied Analysis, vol. 2013, Article ID 42916, 1-17. Wang, S.Q. , Yang, Y. J. and Jassim, H. K., (2014), Local Fractional Function Decomposition Method for Solving Inhomogeneous Wave Equations with Local Fractional Derivative, Abstract and Applied Analysis, vol. 2014, Article ID 176395 ,1-7.

Wazwaz ,A. M.,(2011), Linear and Nonlinear Integral Equations: Methods and Applications, Springer, New York, NY, USA.

Xu ,S. , Ling ,X., Zhao, Y. and Jassim, H. K., (2015) ,A Novel Schedule for Solving the Two-Dimensional Diffusion in Fractal Heat Transfer, Thermal Science, Vol. 19, Suppl. 1 ,99-103.

Yan,S. P., Jafari, H., and Jassim, H. K., (2014) , Local Fractional Adomian Decomposition and Function Decomposition Methods for Solving Laplace Equation within Local Fractional Operators, Advances in Mathematical Physics, vol. 2014, Article ID161580 ,1-7.

Yang (10th reference), X. J.,( 2011), Local Fractional Functional Analysis and Its Applications, Asian Academic publisher Limited, Hong Kong,.

Yang (11th reference),X. J., (2011),Local Fractional Integral Transforms, Progr. In Nonlin. Sci., $4,1-225$.

Yang ,X. J., (2012), Local fractional partial differential equations with fractal boundary problems, Advances in Computational Mathematics and its Applications, vol. 1, no. 1,60-63. 